\title{
Evaluation of Rectal and Skin Temperature Variations of Anesthetised Dogs Undergoing Magnetic Resonance Imaging Diagnosis
}

\author{
Andra DEGAN ${ }^{1 *}$, Ruxandra TUDOR ${ }^{1}$, Ruxandra COSTEA $^{1}$, Dragoș BÎRȚOIU ${ }^{1}$, Mihai SĂVESCU ${ }^{1}$, Alexandru \\ ȘONEA ${ }^{1}$
}

${ }^{1}$ University of Agronomic Science and Veterinary Medicine, Faculty of Veterinary Medicine Bucharest, Romania

*corresponding author: andra.degan@yahoo.com

Bulletin UASVM Veterinary Medicine 76(2)/2019

Print ISSN 1843-5270; Electronic ISSN 1843-5378

doi:10.15835/buasvmcn-vm:2019.0024

\begin{abstract}
:
General anesthesia produces different degrees of central nervous depression and changes in the peripheral circulation, therefore affecting the patient's thermoregulatory mechanism. Moreover, the lack of proper, specially designed equipment for magnetic resonance imaging (MRI) environment monitoring can represent a challenge for the anesthetist. We examined the temperature variations correlated with different anesthetic protocols in dogs that underwent general anesthesia in order to evaluate changes in rectal and distal extremities temperature, before and after anesthesia. This study was conducted at the Faculty of Veterinary Medicine in Bucharest, on 21 dogs that were divided in 3 groups depending on the anesthetic protocol used. First group (B) received butorphanol $(0.2$ $\mathrm{mg} / \mathrm{kg}$, intravenously IV), second group (BK) had butorphanol $(0.2 \mathrm{mg} / \mathrm{kg}$ ) and a low dose of ketamine $(2 \mathrm{mg} / \mathrm{kg})$ IV, and group $3(\mathrm{BM})$ was premedicated with butorphanol $(0.2 \mathrm{mg} / \mathrm{kg})$ and midazolam $(0.2 \mathrm{mg} / \mathrm{kg}) \mathrm{IV}$. All patients were induced with propofol i.v. (3.24 \pm 0.68$)$, intubated and maintained with isoflurane and oxygen. We determined rectal temperature before and right after the end of anesthesia with a digital thermometer and distal extremities temperature with the use of a thermal imaging camera attached to a smartphone. There was no significant difference between the rectal temperature before and after anesthesia within the 3 groups. Patients in group BK had a significant change in skin temperature at the end of anesthesia in all limbs (from $31^{\circ} \mathrm{C}$ to $29.8{ }^{\circ} \mathrm{C}, \mathrm{p}=0.008$ and from $31{ }^{\circ} \mathrm{C}$ to $29.7^{\circ} \mathrm{C}, \mathrm{p}=0.009$ ), respectively). Temperature variations were presented before and at the end of anesthesia, for all the groups especially at skin level. This study revealed that mobile thermal imaging represents a non-invasive technique that is helpful in assessing real time temperature changes in patients undergoing general anesthesia.
\end{abstract}

Keywords: anesthesia, MRI, temperature, thermography

\section{Introduction}

General anesthesia is always required in patients undergoing magnetic resonance imaging in order to ensure the restraint of the animals. The importance of anesthesia is correlated also with the effects of it, most of the anesthetics causing central nervous depression, with major impact on vital organs and metabolism (Jordi et al, 2018). In order to reduce the risks associated with general anesthesia is recommended to initiate a proper monitoring of the patients. Monitoring of vital signs during magnetic resonance imaging can be very challenging due to the fact that equipment and accessories are not designed for use in 
MRI environment (Smith, 2015). Hypothermia represents one of the complications during and after general anesthesia, being considered at the same time the most common perioperative thermal disturbance (Sessler, 2016). In different studies the frequency of hypothermia is described as $97.4 \%$ and $83.6 \%$, respectively, for cats and dogs when hypothermia is defined as $T^{\circ}<38.5^{\circ} \mathrm{C}$ (Rose et al., 2016). However, Amstrong et al. (2005) described normothermia for dogs and cats as $37.8-39.2^{\circ} \mathrm{C}$ (Armstrong et al. 2005), so this prevalence of hypothermia might be an overestimation. Among the complications correlated with a decreased body temperature has been noted: bradycardia, hypotension, arrhythmias, hypoventilation, apnea, hypoxaemia and also a reduction of the metabolism (Khenissi et al., 2017). During general anesthesia the temperature monitoring devices often used are represented by digital thermometers, temperature probes (inserted in the esophagus or rectum), unfortunately in case of using an MRI incompatible temperature probe, the patient can suffers burns, most likely at the tip of the wire. Therefore, during MRI scan the monitoring of animal's temperature is not used (Kruse, 2018). Mammals are classified as homeotherms or warm blooded animals (Reece, 2015) and with the help of thermoregulation process being able to maintain stable internal body conditions regardless the changes of the environment temperature (Morrison et al., 2008). Temperature of the core compartment (deep temperature) is kept higher and in tight regulation compared with the periphery being considered the temperature of clinical interest. However thermoregulatory vasomotor control maintains a gradient between core and periphery of $2-4^{\circ} \mathrm{C}$ (Baldo and Palmer, 2018).

\section{Materials and Methods}

The study was conducted at the Faculty of Veterinary Medicine Bucharest in collaboration with the department of imagistic diagnosis between October 2018 and March 2019. A number of 21 dogs went under general anesthesia in order to perform magnetic resonance imaging examination for either suspicion of intracranium or column pathology. Preanesthetic examination was performed according to ASA status (American Society of Anesthesiologists), patients belonging to an ASA 2 or 3 risk group.
Dogs included in this study were divided into 3 groups depending on the premedication protocol used. The induction of anesthesia was made with propofol IV. All patients were intubated endotracheal and maintained with isoflurane (2 $2.5 \%$ ) in oxygen. In the first group were included patients anesthetized with butorphanol IV at a dose of $0.2 \mathrm{mg} / \mathrm{kg}$, while patients in group 2 and 3 received besides butorphanol $(0.2 \mathrm{mg} / \mathrm{kg})$, a low dose of ketamine at $2 \mathrm{mg} / \mathrm{kg}$, IV, respectively midazolam at $0.2 \mathrm{mg} / \mathrm{kg} \mathrm{IV}$.

During general anesthesia we monitored the oscillimetric non-invasive arterial blood pressure and capnography. Crystalloid solutions were administered at 3-5 ml/kg/hour IV throughout anesthesia.

Due to the lack of special MRI monitoring equipment, the temperature probe couldn't be used on our patients. In order to obtain distal extremities thermal images we used a thermal imaging camera attached to our smart phones. The thermal camera used was a Seek Thermal model designed to connect to an Iphone. The temperature that is able to register is ranged between $-40^{\circ}$ Celsius to $300{ }^{\circ} \mathrm{C}$. The images taken are stored into iOS application called Seek Thermal.

Before starting this study, our thermal camera was compared with a proper thermograph. The only differences registered were in the quality of the image, not in the results. All patients that underwent a general anesthesia for the MRI scan had a rectal temperature taken by a digital thermometer before premedication and at the end of the scan. The values recorded in all 3 groups of dogs were not significantly different. Patients were not covered during anesthesia and no warming device could have been used due to the magnetic field in the room. All dogs were seated on pads. The room temperature was maintained at $21^{\circ} \mathrm{C}$ for all the procedures. We recorded the temperature of the paws for forelimbs and hindlimbs only twice, before the administration of any anesthetic drugs (T0) and right after the close of gaseous anesthetic (T1). The right and left forelimbs, respectively hindlimbs were averaged and subtracted from the initial rectal temperature in order to calculate the delta (rectal minus distal extremity temperature) temperature for each time point. For results interpretation we used descriptive statistics for different characteristics and outcome measures regarding the patient, variables like mean +/- 
Table 1. Demographic of the dogs population

\begin{tabular}{cccc}
\hline Variable & $\begin{array}{c}\text { Butorphanol } \\
\text { group }=\mathbf{7}\end{array}$ & $\begin{array}{c}\text { Butorphanol + } \\
\text { Ketamine }=7\end{array}$ & $\begin{array}{c}\text { Butorphanol + } \\
\text { Midazolam }=7\end{array}$ \\
\hline Age (years)* & $5.9 \pm 4.19$ & $7.14 \pm 3.07$ & $8.42 \pm 1.27$ \\
\hline Weight ( kg)* & $6.05 \pm 2.03$ & $8.18 \pm 2.83$ & $10.08 \pm 4.28$ \\
\hline Propofol dose (mg/kg)* & $3,17 \pm 0.23$ & $3,14 \pm 0.89$ & $3.42 \pm 0.78$ \\
\hline $\begin{array}{c}\text { Time of anesthesia } \\
\text { (min)* }\end{array}$ & $42.8 \pm 9.5$ & $47.8 \pm 6.3$ & $52.1 \pm 12.1$ \\
\hline *Variable represent & &
\end{tabular}

*Variables represent the mean \pm standard deviation

Table 2. Temperature variations of the limbs

\begin{tabular}{ccccc}
\hline \multicolumn{5}{c}{ Temperature* ${ }^{0}$ Celsius) } \\
\hline Group & FLB & FLA & HLB & HLA \\
\hline $\begin{array}{c}\text { Butorphanol } \\
\text { Butorphanol } \\
+ \text { Ketamine }\end{array}$ & $31.6 \pm 3$ & $31.4 \pm 1.88$ & $30.7 \pm 3.98$ & $30.9 \pm 0.97$ \\
\hline $\begin{array}{c}\text { Butorphanol }+ \\
\text { Midazolam }\end{array}$ & $30.8 \pm 2.83$ & $29.8 \pm 3.64$ & $31 \pm 3.30$ & $29.7 \pm 3.32$ \\
\hline
\end{tabular}

*Values represent the mean \pm standard deviation of limbs temperature before and after anesthesia

$\mathrm{FLB}=$ forelimbs before anesthesia; FLA=forelimbs after anesthesia

$\mathrm{HLB}=$ hindlimbs before anesthesia; HLA= hindlimbs after anesthesia

standard deviation, percentage. $\mathrm{P}$ values were used to determine the difference between rectal temperature and the average and delta distal extremities temperature.

\section{Results and discussions}

The total of dogs that underwent general anesthesia for MRI scan was 21. Our patients ages ranged from 0.3 years to 12 years old (mean 7.15 years old). Dogs in our study weigh between 3.1 and $17.3 \mathrm{~kg}$ (mean 8,1 $\pm 7,6$ ). The total dose of propofol used for induction of anesthesia was not significantly different between our groups and ranged from 2 to $5 \mathrm{mg} / \mathrm{kg}$ with a mean and standard deviation of $3.24 \pm 0.68$. The duration of anesthesia was $47.6 \pm 9.9$ minutes.

We calculated delta temperature by subtracting the average thermal temperatures of either forelimbs or hindlimbs from the initial rectal temperature at each time point. There were no significant differences between initial rectal temperature and the distal extremities temperatures in all the groups, at each time point.

In patients premedicated with butorphanol and ketamine we remarked a significantly change in distal extremities temperatures, for both fore and hindlimb, before premedicatioan and right after the close of isoflurane.

The results obtained showed a decrease in forelimbs peripheral temperature (before and after anesthesia) from $31^{\circ} \mathrm{C}$ to $29.8^{\circ} \mathrm{C}(\mathrm{p}=0.008)$ and from $31^{\circ} \mathrm{C}$ to $29.7^{\circ} \mathrm{C}(\mathrm{p}=0.009)$ for hindlimbs respectively.

There were no changes in the other 2 groups regarding the difference between the initial peripheral temperature in both forelimbs and hindlimbs, and the perihperal temperature at the end of anesthesia.

However, significantly differences were also recorded in the delta temperature (the initial rectal temperature minus distal extremity temperature) of forelimbs ( $p=0.006$; from $7.6^{\circ} \mathrm{C}$ to $\left.8.9^{\circ} \mathrm{C}\right)$ and hindlimbs $\left(p=0.007\right.$; from $7.8^{\circ} \mathrm{C}$ to $\left.9^{\circ} \mathrm{C}\right)$ between the two time point (before and after anesthesia) in patients premedicated with butorphanol and ketamine.

In this study we observed a change in the peripheral temperature of both fore and hindlimbs in dogs that underwent a general anesthesia for an MRI scan. The only significant difference was seen in patients premedicated with butorphanol and ketamine. Dogs in this group suffered a decrease between the average peripheral limb temperature before and at the end on anesthesia, compared with patients premedicated with butorphanol and midazolam or only with butorphanol.

Different studies describe the effects of injectable anesthetics like propofol or volatile anesthetic gases on the body temperature in human medicine. It has been shown that propofol 

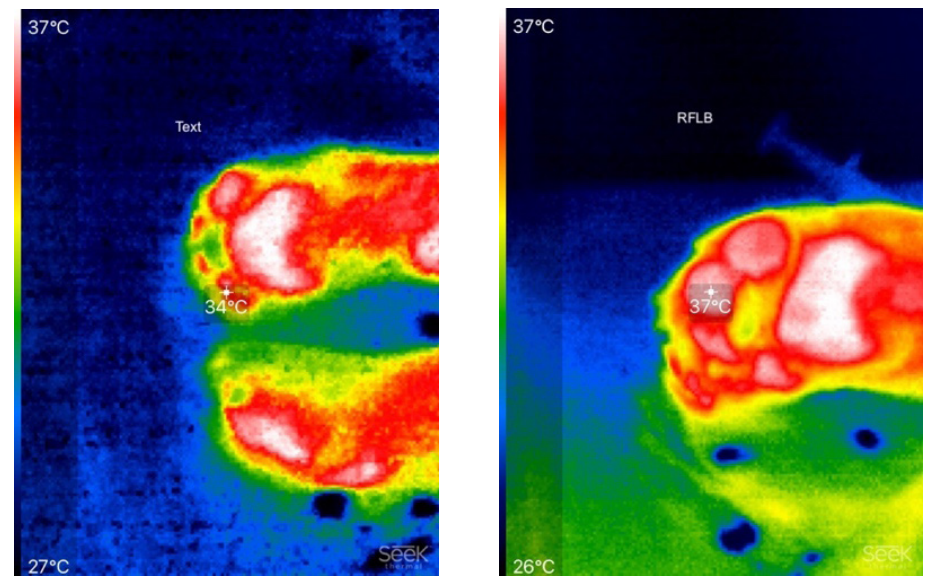

Figure 3A. Left and right forelimbs before premedication of anesthesia in a patient anesthesized with butorphanol and ketamine.
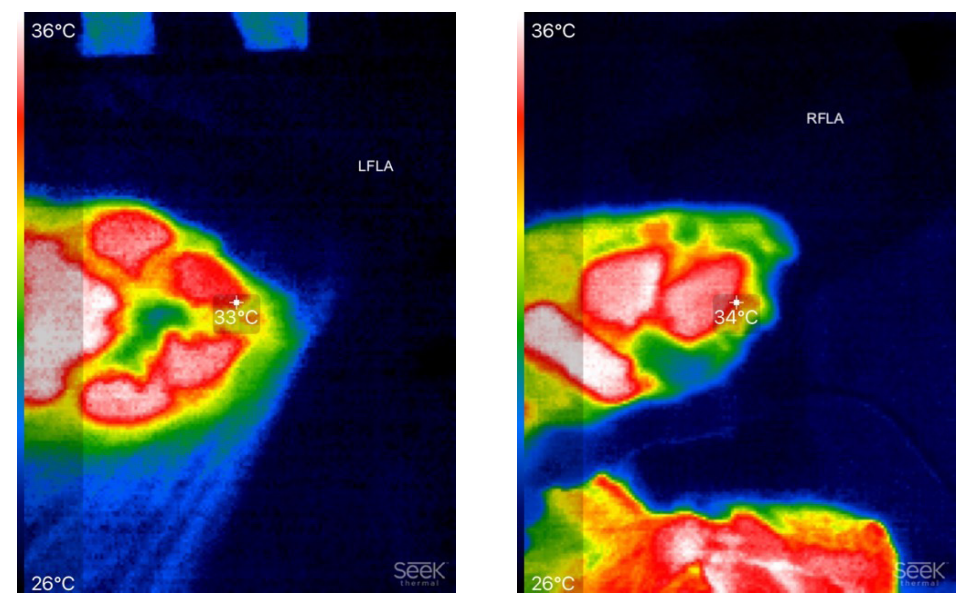

Figure 3B. Left forelimb and right forelimb in the same patient as in figure 1A. The images were taken right after the close of isoflurane

determined a decrease in body surface temperature with different values depending on the time that passed since propofol administration. Right after the administration the authors have seen a drop in temperature with a reversible effect and an increase as soon as the propofol was discontinued and the effect has passed (Rajasekaran et al., 2018). Both, propofol and volatile anesthetic gases have similar mechanisms on tissues perfusion, by inhibiting the sympathetic vasoconstrictive nerve cells and resulting in vessel dilation and thermal radiation (Noguchi et al., 2002).

In a previous report (Posner et al, 2010), cats premedicated with opioids had an increase in body temperature. The combination of hydromorphone with ketamine determined a mild hyperthermia, but a higher dose of ketamine (20 $\mathrm{mg} / \mathrm{kg}$ ) produced a decrease in body temperature by $\sim 1.6^{\circ} \mathrm{C}$ (Beck et al., 1971).

This technique was used by researches in order to evaluate extremity perfusion in patients with vascular diseases (Wallace et al., 2018). Continues thermal imagery offers the potential to monitor real time therapeutic response to vasoactive agents to improve peripheral tissue perfusion or to immediately discontinue ineffective modalities in other clinical settings (Kelechi and Michel, 2007; Wallace et al., 2018).

For this study we decided to use the technique due to the lack of body temperature determinations during anesthesia in patients undergoing MRI scan. It provided us a real time monitoring of peripheral temperature by converting the thermal emission field into a single image with temperature points, recorded into a fraction of a second. The 

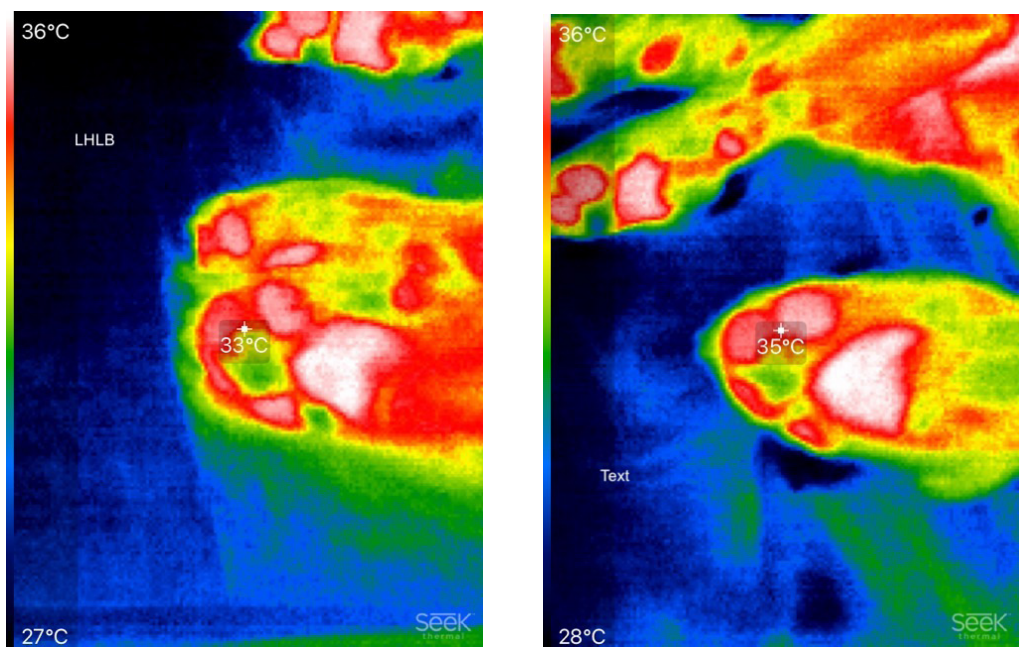

Figure $4 \mathrm{~A}$. Left and right hindlimbs distal extremities of the same patient as in figure $1 \mathrm{~A}$ and B, before the administration of premedication.
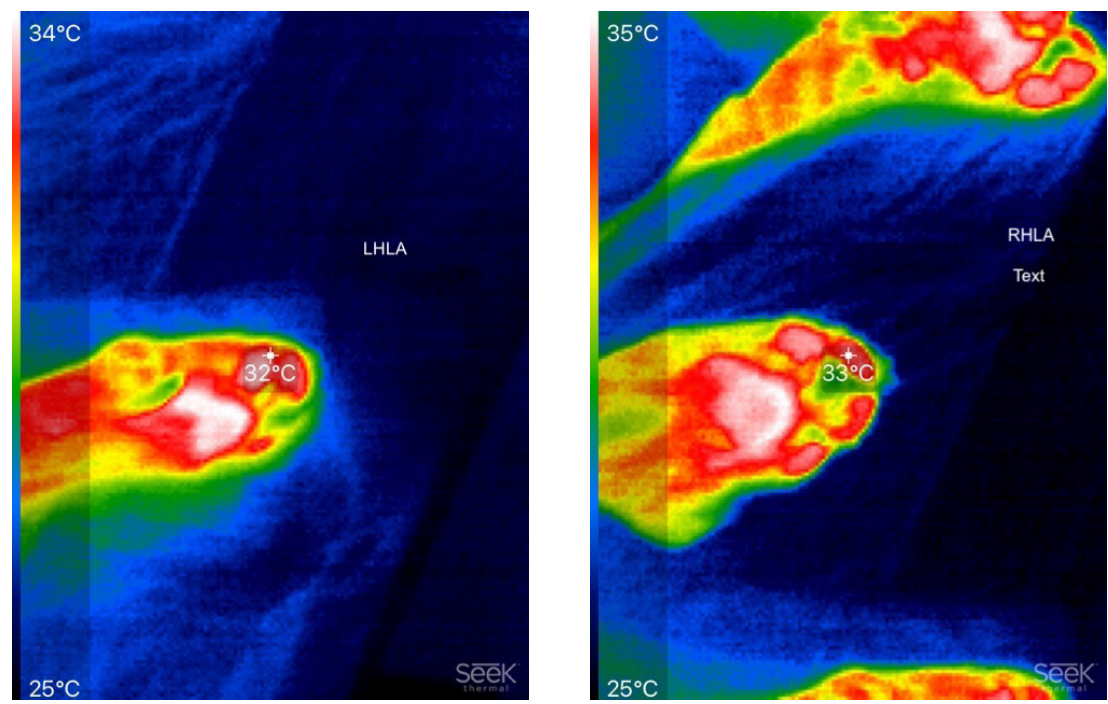

Figure 4 B. Temperature of left and right hindlimbs distal extremities (paws) at the end of anesthetic procedure.

advantages of a thermal mobile camera are the little training required and that it doesn't come in direct contact with the patients.

The study does have limitations due to the only two time points when temperature was determined, with no data on body or peripheral temperature during anesthesia. Moreover, compared with other studies, we used for our patients different anesthetics such as propofol and isoflurane, drugs that are able to influence temperature. However there was no significant difference in propofol doses between the three groups. Also patients that underwent general anesthesia had neurological disease, either intracranial $(n=7)$ or column diseases $(n=14)$ that can affect the thermoregulation mechanism. All these patients had a uniform division between the three groups. Another limitation can be represented by the different body surface and the coat of the patients belonging to different breeds.

\section{Conclusions}

Our study demonstrates the feasibility of using a mobile thermal imager to asses temperature changes in patients undergoing general anesthesia. The use of a mobile thermal imager to determine skin temperature can serve as a tool for hemodynamic monitoring in sedated animals. There were no significant changes in rectal and skin peripherial temperature in group B and BM, 
however dogs premedicated with butorphanol and ketamine had a significant decrease between the two time points, regarding the peripheral skin temperature.

Acknowledgments. This research did not receive any grant from funding agencies in the public, commercial or not-for-profit sectors.

\section{References}

1. Armstrong SR, Roberts BK, Aronsohn M (2005). Perioperative hypothermia. Journal of Veterinary Emergency and Critical Care, 15(1):32-37.

2. Baldo C, Palmer D (2018). Temperature Regulation and Monitoring. Veterinary Anesthetic and Monitoring Equipment, 285-302.

3. Beck CC, Coppock RW, Ott BS (1971). Evaluation of Vetalar (ketamine $\mathrm{HCl}$ ). A unique feline anesthetic. Veterinary medicine, small animal clinician: VM, SAC, 66(10):993.

4. Smith JA (2015). Patient and Anesthetist Safety Considerations for Laser and Radiographic Procedures and Magnetic Resonance Imaging. Veterinary Anesthesia and Analgesia: The Fifth Edition of Lumb and Jones, 10151026

5. Kelechi TJ, Michel Y (2007). A descriptive study of skin temperature, tissue perfusion, and tissue oxygen in patients with chronic venous disease. Biological research for nursing, 9(1):70-80.

6. Khenissi L, Covey-Crump G, Knowles TG, Murrell J (2017). Do heat and moisture exchangers in the anaesthesia breathing circuit preserve body temperature in dogs undergoing anaesthesia for magnetic resonance imaging? Veterinary anesthesia and analgesia, 44(3):452460.

7. Kruse EK (2018). Equipment for the Magnetic Resonance Imaging System. Veterinary Anesthetic and Monitoring Equipment, 335-348.

8. Morrison SF, Nakamura K, Madden CJ (2008). Central control of thermogenesis in mammals. Experimental physiology, 93(7):773-797.

9. Noguchi I, Matsukawa T, Ozaki M, Amemiya Y (2002). Propofol in low doses causes redistribution of body heat in male volunteers. European journal of anaesthesiology, 19(9):677-681.

10. Rajasekaran S, Pressler M, Parker J, Scales A, Andersen $\mathrm{N}$, Olivero A, McGough R (2018). Quantifying and Trending the Thermal Signal as an Index of Perfusion in Patients Sedated with Propofol. Multidisciplinary Digital Publishing Institute. Healthcare, 6(3):87.

11. Reece WO (2015). Body temperature and its regulation. in Dukes' Physiology of Domestic Animals, 149.

12. Rose N, Kwong GP, Pang DS (2016). A clinical audit cycle of post operative hypothermia in dogs. Journal of Small Animal Practice, 57(9):447-452.

13. Sessler DI (2016). Perioperative thermoregulation and heat balance. The Lancet, 387(10038), 2655-2664.

14. Tremoleda JL, Macholl S, Sosabowski JK (2018). Anesthesia and monitoring of animals during MRI studies. In Preclinical MRI (pp. 423-439). Humana Press, New York, NY.

15. Wallace GA, Singh N, Quiroga E, Tran NT (2018). The use of smart phone thermal imaging for assessment of peripheral perfusion in vascular patients. Annals of vascular surgery, 47:157-161. 